\title{
Challenges of cardiac inflammation imaging with F-18 FDG positron emission tomography
}

\author{
Pradeep Bhambhvani, $M^{a}$ \\ ${ }^{a}$ Department of Radiology, Division of Molecular Imaging and Therapeutics, The University of \\ Alabama at Birmingham, Birmingham, AL
}

Received Apr 7, 2016; accepted Apr 7, 2016

doi: 10.1007/s12350-016-0508-1

\section{See related article, pp. 86-99}

Reliable hot spot inflammation imaging with fluorine18 fluorodeoxyglucose (FDG) positron emission tomography (PET) in suspected cardiac infection, cardiac sarcoidosis, or vulnerable coronary plaque is only possible when physiologic myocardial FDG uptake can be adequately suppressed. The gamut of perfusion-metabolism patterns possible in cardiac sarcoidosis ${ }^{1}$ combined with the erratic and real possibility of inadequate suppression of normal myocardial FDG uptake is a sure recipe for head scratching and challenging image interpretation. A number of preparation methods aimed at suppressing physiologic myocardial glucose utilization have been described without clear consensus on the optimal method. In the present issue of the Journal of Nuclear Cardiology, Osborne and colleagues (doi:10.1007/s12350-016-0502-7) review various preparation protocols and success rates achieved in published studies for FDG cardiac PET and provide useful practical recommendations.

A brief overview of cardiomyocyte metabolism is essential to appreciate the challenges and rationale of interventions. The heart uses various substrates as energy sources including free fatty acids (FFAs), glucose, and lactate. In the fasting condition, the normal myocardium utilizes FFAs as the major energy source (90\%). Other sources include glucose and lactate. ${ }^{2}$ Postmeals or in the dysfunctional myocardium (example ischemic heart disease), myocardial metabolism shifts to

Reprint requests: Pradeep Bhambhvani, MD, Department of Radiology, Division of Molecular Imaging and Therapeutics, The University of Alabama at Birmingham, Birmingham, AL; pbhambhvani@uabmc.edu

J Nucl Cardiol 2017;24:100-2.

$1071-3581 / \$ 34.00$

Copyright (C) 2016 American Society of Nuclear Cardiology. glucose per the glucose-fatty acid cycle. ${ }^{2,3}$ Elevated blood insulin and glucose levels with decreased FFAs lead to a relative rise in myocardial glucose consumption. In contrast, during fasting, there is an increase in FFAs with decrease in insulin and glucose levels, shifting myocardial energy consumption away from glucose and toward FFAs. Hence interventions that facilitate myocardial FFAs metabolism while at the same time suppress physiologic glucose metabolism are imperative for successful FDG PET cardiac inflammation imaging. These include avoidance of strenuous exercise prior to imaging (to prevent skeletal muscle glucose utilization), high-fat with low (less than $5 \mathrm{~g}$ )- or no carbohydrate diet (to increase blood FFAs and minimize glucose and insulin levels), addition of a high-fat drink to this diet prior to FDG injection (to elevate blood FFAs), fasting for 4-18 hours (to decrease blood glucose and insulin levels and increase FFAs), and certain pharmacologic maneuvers. The latter include unfractionated heparin which induces lipolysis and up to fivefold increase in blood FFAs ${ }^{4}$ and calcium channel blockers which reduce myocardial FDG uptake. ${ }^{5}$ Most studies combine two or more interventions with the goal of complete suppression of normal cardiac FDG uptake.

Tang and colleagues in a meta-analysis involving 16 studies and 559 patients evaluated for cardiac sarcoidosis examined various patient preparations that impact the diagnostic performance of FDG PET. They conclude that duration of fasting and heparin administration significantly affected the diagnostic odds ratio $(P=.01$ and .04 , respectively), whereas a high fat low cholesterol diet did not have a significant effect $(P=.17) .{ }^{6}$ Nonspecific findings that may indicate failed suppression, and/or physiologic uptake and make image interpretation challenging included diffuse myocardial activity exceeding liver uptake or uptake in the lateral wall and/or ring shaped/circumferential basal uptake (Nensa et al doi: $10.1007 / \mathrm{s} 12350-016-0443-1)^{7}$ or papillary muscle uptake. ${ }^{8,9}$ Diffuse FDG uptake in the entire left ventricular 
wall without focal uptake generally does not indicate disease as cardiac sarcoidosis is histopathologically known to be localized and not diffuse. ${ }^{10}$ Absence of contrast enhancement, edema, or wall motion abnormalities on cardiac MRI associated with these nonspecific FDG uptake patterns is useful in avoiding false positive interpretations (Nensa et al doi:10.1007/s12350-016-0443-1). ${ }^{8}$

Based on comprehensive literature review and their institutional experience, Osborne and colleagues (doi: 10.1007/s12350-016-0502-7) recommend the use of at least two high-fat and no carbohydrate (HFNC) meals (for example at dinner the night before and breakfast on day of scan) followed by a fast of at least four hours before FDG injection as the most effective preparation method for cardiac FDG PET inflammation imaging. Another protocol involving unfractionated heparin administered 15 minutes before FDG injection and following at least one high-fat and low-carbohydrate (HFLC) meal (example at dinner the night before scan) and an overnight fast is equally effective but requires exposure to an intravenous medication with possible adverse effects. They do not recommend the fasting only preparation method with exception for those who cannot eat or have dietary restrictions precluding the HFNC or HFLC diets and suggest fasting for at least 18 hours in such individuals. Vigorous exercise should be avoided for 24 hours prior to minimize skeletal muscle uptake. Furthermore, the authors do not advocate the use of calcium channel blockers, unrestricted diets, and eating or drinking anything including high-fat supplements within at least four hours of the test. The authors believe adherence to the above recommendations should result in myocardial FDG suppression sufficient for diagnostic evaluation in greater than $80 \%$ of studies.

From the 31 different dietary interventions with or without heparin/verapamil premedication reviewed by Osborne et al, optimal suppression of myocardial FDG uptake was noted in $87 \%-93 \%$ of patients prepared with two HFNC meals and 4 hour fast ${ }^{1,8}$ leading them to recommend it as the most effective preparation method. Strategies using intravenous heparin at a dose of $50 \mathrm{IU} \cdot \mathrm{kg}^{-1} 15$ minutes prior to FDG injection in combination with either a low-carbohydrate diet +12 hour fast $^{11}$ or 18 -hour fasting with low-carbohydrate diet ${ }^{12}$ led to adequate suppression in $88 \%-100 \%$ of patients. Other investigators have questioned the efficacy of heparin. ${ }^{7,13}$ Coulden et al reported successful myocardial suppression in 92 out of 94 oncology patients (98\%) who were compliant with only an "Atkins style" lowcarbohydrate diet (less than $3 \mathrm{~g}$ ) the day before examination followed by an overnight fast. ${ }^{14} 26$ out of 120 $(22 \%)$ patients were noncompliant with the "Atkins style" diet. While the shorter duration of fasting (46 hours) after the two HFNC meals as recommended by
Osborne and colleagues should lead to improved compliance, the preceding no or low-carbohydrate diet could, however, result in carbohydrate indiscretions in the less motivated/informed patient. Since their review, Nensa et al (doi:10.1007/s12350-016-0443-1) have shown homogeneous suppression of myocardial FDG uptake in 79/94 (84\%) patients prepared with a $\geq 24$ hour high-fat, low-carbohydrate, protein-permitted diet without fasting and intravenous injection of unfractionated heparin $\left(50 \mathrm{IU} \cdot \mathrm{kg}^{-1}\right) 15$ minutes prior to FDG administration using cardiac PET/MR.

The pharmacologic interventions merit further discussion. In 1979, Asmal et al demonstrated the anticoagulant and lipolytic actions of incremental doses of heparin in five healthy volunteers. ${ }^{4}$ They noted a significant anticoagulant effect (measured by partial thromboplastin time) after intravenous heparin administration at a dose of $15 \mathrm{U} \cdot \mathrm{kg}^{-1}$ and no significant change in partial thromboplastin time over the control value $(36 \pm 2$ seconds) with lower doses, i.e., 5 and $10 \mathrm{U} \cdot \mathrm{kg}^{-1}$ respectively $(P>.1)$. FFAs were significantly higher after the 5 and $10 \mathrm{U} \cdot \mathrm{kg}^{-1}$ doses $(P<.0025)$ with further increments after higher heparin doses. Asmal et al clearly illustrate the significant lipolytic action without significant anticoagulant effects with both the 5 and $10 \mathrm{U} \cdot \mathrm{kg}^{-1}$ heparin doses. The published cardiac FDG PET studies with unfractionated heparin that were successful in suppressing myocardial FDG uptake have been done at higher anticoagulant doses i.e., $50 \mathrm{U} \cdot \mathrm{kg}^{-1}$ intravenously ${ }^{7,11,12}$ etc. To overcome the potentially detrimental anticoagulant effect while maintaining desired lipolysis as observed by Asmal et al, studies evaluating the effectiveness of myocardial FDG suppression with lower doses of unfractionated heparin (for example 5-10 U.kg ${ }^{-1}$ ) in combination with dietary preparation are much needed. In addition to bleeding, another safety concern with heparin use is the uncommon but potentially life threatening risk of heparin-induced thrombocytopenia (HIT) and thrombosis. No dose of heparin is too low to cause HIT, as even small doses used for intravenous catheter flushes have been associated with HIT. ${ }^{15,16}$ In a meta-analysis, the absolute risk for HIT with low molecular weight heparin was $0.2 \%$ (95\% CI $0.1 \%$ $0.4 \%)$, and with unfractionated heparin was $2.6 \%$ (95\% CI $1.5 \%-3.8 \%) .{ }^{17}$ The lower risk of developing HIT with low molecular weight heparin is countered by its inferior lipolytic effects when compared to unfractionated heparin. ${ }^{18}$ While Persson et al showed superior lipolysis with unfractionated heparin (fivefold increase in FFAs); they also noted a threefold rise in FFAs with low molecular weight heparin compared to control. ${ }^{18}$ This observation along with a lower incidence of HIT presents a research opportunity into the lipolytic effects of low molecular weight heparin in combination with HFNC/HFLC diet prior to FDG cardiac PET. 
In a mouse model, Gaeta and colleagues showed significant reduction in myocardial FDG uptake following single administration of verapamil injected 1 hour prior to FDG administration at doses of 1 and $20 \mathrm{mg} \cdot \mathrm{kg}^{-1}$. 5 Similar results have not been reproduced in humans. Demeure and colleagues demonstrated similar success in suppressing myocardial FDG uptake (89\%) in 9 volunteers pre-treated with oral Verapamil $(120 \mathrm{mg})$ 1 hour before FDG injection who also ate a high-fat lowcarbohydrate meal, followed by a 12-h fasting period when compared to 9 other volunteers who only ate a highfat low-carbohydrate meal, followed by a 12-hour fasting period without verapamil premedication. ${ }^{19}$

In conclusion, since patient preparation has such an important role in the determining cardiac FDG PET image quality, the dietary, fasting, and exercise recommendations should be specified in a detailed information sheet, reviewed with the patient and/or caregiver, and reinforced prior to scan date and confirmed for compliance on day of scan. Any deviation from written patient instructions should result in study cancelation and rescheduling to avoid the possibility of inadequate suppression of normal cardiac FDG uptake. Nonspecific incomplete suppression patterns including diffuse myocardial uptake or lateral wall or basal ring distribution activity or papillary muscle activity despite adequate preparation can hamper image analysis and may need further characterization with MRI to exclude false positive interpretation. With obvious exceptions of claustrophobia, incompatible devices, contrast allergy, and renal dysfunction, the positive attributes of the two modalities dovetail nicely for hybrid cardiac PET/MR inflammation imaging. Further investigation into the lipolytic efficacy of the potentially safer lower doses of unfractionated heparin $\left(5-10 \mathrm{U} \cdot \mathrm{kg}^{-1}\right)$ and low molecular weight heparin is in order as well. Osborne et al rightfully point to the possibility of incomplete suppression of physiologic myocardial glucose uptake in a small proportion of studies despite all interventions and call for a continuous review of image quality and protocol tweaking as needed with a goal of $80 \%$ adequacy, as well as the development of alternate and more specific inflammation imaging radiotracers.

\section{References}

1. Blankstein R, Osborne M, Naya M, Waller A, Kim CK, Murthy VL, et al. Cardiac positron emission tomography enhances prognostic assessments of patients with suspected cardiac sarcoidosis. J Am Coll Cardiol 2014;63:329-36.

2. Yoshinaga K, Tamaki N. Imaging myocardial metabolism. Curr Opin Biotechnol 2007;18:52-9.

3. Randle PJ, Garland PB, Hales CN, Newsholme EA. The glucose fatty-acid cycle. Its role in insulin sensitivity and the metabolic disturbances of diabetes mellitus. Lancet 1963;1:785-9.
4. Asmal AC, Leary WP, Thandroyen F, Botha J, Wattrus S. A doseresponse study of the anticoagulant and lipolytic activities of heparin in normal subjects. Br J Clin Pharmacol 1979;7:531-3.

5. Gaeta C, Fernandez Y, Pavia J, Flotats A, Artigas C, Deportos J, et al. Reduced myocardial 18F-FDG uptake after calcium channel blocker administration. Initial observation for a potential new method to improve plaque detection. Eur J Nucl Med Mol Imaging 2011:38:2018-24.

6. Tang R, Wang JT, Wang L, Le K, Huang Y, Hickey AJ et al. Impact of patient preparation on the diagnostic performance of $18 \mathrm{~F}-\mathrm{FDG}$ PET in cardiac sarcoidosis: A systematic review and meta-analysis. Clin Nucl Med 2015. doi:10.1097/RLU.0000000000001063.

7. Morooka M, Moroi M, Uno K, Ito K, Wu J, Nakagawa T, et al. Long fasting is effective in inhibiting physiological myocardial 18F-FDG uptake and for evaluating active lesions of cardiac sarcoidosis. EJNMMI Res 2014;4:1.

8. Soussan M, Brillet PY, Nunes H, Pop G, Ouvrier MJ, Naggara N, et al. Clinical value of a high-fat and low-carbohydrate diet before FDG-PET/CT for evaluation of patients with suspected cardiac sarcoidosis. J Nucl Cardiol 2013;20:120-7.

9. Maurer AH, Burshteyn M, Adler LP, Steiner RM. How to differentiate benign versus malignant cardiac and paracardiac 18F FDG uptake at oncologic PET/CT. Radiographics 2011;31:1287-305.

10. Ishida Y, Yoshinaga K, Miyagawa M, Moroi M, Kondoh C, Kiso $\mathrm{K}$, et al. Recommendations for (18)F-fluorodeoxyglucose positron emission tomography imaging for cardiac sarcoidosis: Japanese Society of Nuclear Cardiology recommendations. Ann Nucl Med 2014;28:393-403.

11. Scholtens AM, Verberne HJ, Budde RP, Lam M. Additional heparin pre-administration improves cardiac glucose metabolism suppression over low carbohydrate diet alone in 18F-FDG-PET imaging. J Nucl Med 2016;57(4):568-73.

12. Manabe O, Yoshinaga K, Ohira H, Masuda A, Sato T, Tsujino I, et al. The effects of 18-h fasting with low-carbohydrate diet preparation on suppressed physiological myocardial (18)F-fluorodeoxyglucose (FDG) uptake and possible minimal effects of unfractionated heparin use in patients with suspected cardiac involvement sarcoidosis. J Nucl Cardiol 2016;23:244-52.

13. Gormsen LC, Christensen NL, Bendstrup E, Tolbod LP, Nielsen SS. Complete somatostatin-induced insulin suppression combined with heparin loading does not significantly suppress myocardial 18F-FDG uptake in patients with suspected cardiac sarcoidosis. $\mathbf{J}$ Nucl Cardiol 2013;20:1108-15.

14. Coulden R, Chung P, Sonnex E, Ibrahim Q, Maguire C, Abele J. Suppression of myocardial 18F-FDG uptake with a preparatory "Atkins-style" low-carbohydrate diet. Eur Radiol 2012;22:2221-8.

15. Heeger PS, Backstrom JT. Heparin flushes and thrombocytopenia. Ann Intern Med 1986;105:143.

16. Muslimani AA, Ricaurte B, Daw HA. Immune heparin-induced thrombocytopenia resulting from preceding exposure to heparin catheter flushes. Am J Hematol 2007;82:652-5.

17. Martel N, Lee J, Wells PS. Risk for heparin-induced thrombocytopenia with unfractionated and low-molecular-weight heparin thromboprophylaxis: A meta-analysis. Blood 2005;106:2710-5.

18. Persson E, Nordenstrom J, Nilsson-Ehle P, Hagenfeldt L, Wahren J. Plasma lipolytic activity and substrate oxidation after intravenous administration of heparin and a low molecular weight heparin fragment. Clin Physiol 1990;10:573-83.

19. Demeure F, Hanin FX, Bol A, Vincent MF, Pouleur AC, Gerber B, et al. A randomized trial on the optimization of $18 \mathrm{~F}-\mathrm{FDG}$ myocardial uptake suppression: Implications for vulnerable coronary plaque imaging. J Nucl Med 2014;55:1629-35. 